\title{
Shipworm symbiosis ecology-guided discovery of an antibiotic that kills colistin-resistant Acinetobacter
}

\author{
Bailey W. Miller, ${ }^{[a]}$ Albebson Lim, ${ }^{[a]}$ Zenjian Lin, ${ }^{[a]}$ Jeannie Bailey, ${ }^{[b]}$ Mark A. Fisher ${ }^{[c]}$ Louis R. \\ Barrows, ${ }^{[\mathrm{d}]}$ Colin Manoil, ${ }^{[\mathrm{b}]}$ Eric W. Schmidt, ${ }^{*[\mathrm{a}]}$ and Margo G. Haygood ${ }^{*[a]}$
}
[a] Dr. B.W. Miller, A. Lim, Dr. Z. Lin, Dr. E.W. Schmidt, and Dr. M.G. Haygood Department of Medicinal Chemistry
University of Utah
30 S. 2000 E., Salt Lake City, UT 84112, United States of America
E-mail: ews1@utah.edu, margo.havgood@utah.edu
[b] Dr. J. Bailey, Dr. C. Manoil
Department of Genome Sciences
University of Washington
Seattle, Washington, United States of America
[c] Dr. M.A. Fisher
Department of Pathology and ARUP Laboratories
University of Utah
Salt Lake City, UT 84112, United States of America
[d] Dr. L.R. Barrows
Department of Pharmacology and Toxicology
University of Utah
Salt Lake City, UT 84112, United States of America

Supporting information for this article is given via a link at the end of the document.

Abstract: Teredinibacter turnerae is an intracellular bacterial symbiont that lives in the gills of wood-eating shipworms, where it is proposed to use antibiotics to defend itself and its animal host. Several biosynthetic gene clusters are conserved in $T$. turnerae and in their host shipworms around the world, implying that they encode the important defensive antibiotics. Here, we describe turnercyclamycins, lipopeptide antibiotics encoded in the genomes of all sequenced $T$. turnerae strains. Turnercyclamycins A and B are bactericidal against challenging Gram-negative pathogens, Escherichia coli, Klebsiella pneumoniae, and colistin-resistant Acinetobacter baumannii, at 1, 2, and $8 \mu \mathrm{g} / \mathrm{mL}$, respectively, while lacking toxicity to mammalian cells. Phenotypic screening identified the outer membrane as the likely target. By exploring the inhabitants of environments that select for the properties we require, we can harvest the fruits of evolution to discover compounds with potential to target unmet health needs. Investigating the symbionts of animals, and shipworms in particular, is a powerful example of this principle.

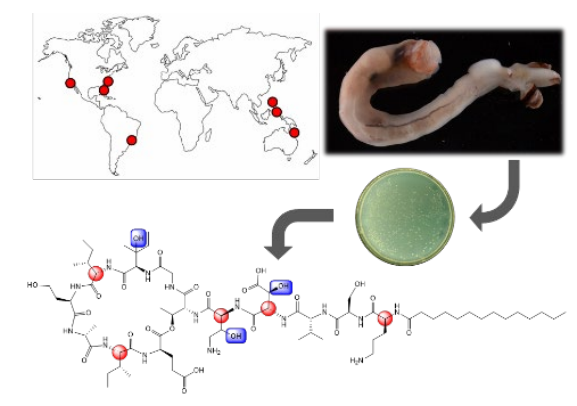

\section{Introduction}

Wood eating shipworms (Teredinidae), a family of marine bivalve mollusks, have a close symbiotic relationship with the intracellular bacteria living in their gills. These bacteria, largely represented by the gammaproteobacterial genus Teredinibacter, support their hosts both through the fixation of atmospheric nitrogen and by contributing cellulolytic enzymes necessary for wood degradation. ${ }^{[1-3]}$ While these microbes are located in bacteriocytes within the gill filaments of the shipworm, their enzymes are transported to and can be detected in the cecum, where wood is digested..$^{[4]}$ Interestingly, this organ has been shown to be nearly devoid of bacterial inhabitants, despite a presumed abundance of nutritionally accessible carbon released by digested lignocellulose. ${ }^{[5]}$ This observation led to the hypothesis that the symbiotic Teredinibacter cells are also producing antimicrobial secondary metabolites in order to both protect their ecological niche within the gill tissues and suppress pathogenic or opportunistic microbes in the cecum to protect their host's food 
source. ${ }^{[6,7]}$ If so, the symbiosis might provide a ready source of new antibiotics to target the rise of multidrug resistance, a crucial problem in human health.

Typically, intracellular symbiotic bacteria involved in nutritional symbioses are difficult to cultivate, which limits access to isolatable quantities of ecologically important metabolites. However, a number Teredinibacter turnerae strains have been successfully cultured, facilitating genome sequencing and large scale fermentation for natural product isolation. The most well characterized representative of this group, T. turnerae T7901, was identified as an excellent potential source of secondary metabolites based on the high content of complex polyketide synthase (PKS) and nonribosomal peptide synthetase (NRPS) biosynthetic gene clusters. ${ }^{[7]} \mathrm{A}$ recent systematic analysis of additional cultivated symbiont genomes and shipworm gill metagenomes uncovered a vast pool of uncharacterized biosynthetic gene clusters (BGCs), including five PKS and/or NRPS-containing clusters that are found in all genomes of cultivated $T$. turnerae and in all metagenomes of shipworms that harbor $T$. turnerae as major symbionts.(8) These symbionts and hosts were collected from around the world, including tropical and temperate waters, implying that the compounds produced by the BGCs are important for the symbiotic relationship. ${ }^{[8]}$

Of the five PKS/NRPS BGCs, only two produce compounds with known structures, both of which were isolated from $T$. turnerae T7901 and then detected in shipworm tissues. One BGC encodes the triscatecholate siderophore turnerbactin. ${ }^{[9]}$ Siderophores are ecologically important compounds for both free living microbes and those in symbiotic systems. ${ }^{[10]} \mathrm{A}$ second BGC encodes the boronated polyketide macrolides tartrolon D/E, which are potently active against apicomplexan parasites with low picomolar activity against some strains, but lack toxicity to human cell lines. ${ }^{[11]}$ These data supported the hypothesis that the ecology of shipworm symbiosis leads to the synthesis of biologically active and ecologically relevant molecules, driving us to seek the potential remaining, conserved antibiotics encoded in T. turnerae genomes.

Herein, we describe the identification of the chemical products from one of the most common shipworm BGCs as potent antibiotics. The turnercyclamycins represent a new family of lipopeptides that kill several challenging Gram-negative bacterial species, including Acinetobacter baumannii, which has been designated by the World Health Organization as one of the most successful and serious of the ESKAPE pathogens, ${ }^{[12]}$ and yet are not toxic to mammalian cells. Colistin is the last-line therapy for otherwise lethal $A$. baumannii infections, but increasing instances of resistance threaten to leave the disease without viable treatment options. Here, we show that turnercyclamycins target the outer membrane like colistin, but they retain efficacy against colistin-resistant Acinetobacter, making them promising leads for antimicrobial development.

The turnercyclamycins were not previously observed in $T$. turnerae T7901 cultures in part because the crude mixture of turnercyclamycins is nearly insoluble in common solvents used in natural products purification methods, although they are well behaved when purified. Bioinformatics analysis of the tur pathway to turnercyclamcyins reveals that the pathway is very highly conserved across shipworm isolates and gills, so that exactly the same suite of compounds should be found in $T$. turnerae-containing shipworms globally. These results reinforce the utility of symbiosis and chemical ecology in providing new solutions to multidrug resistant infections.

\section{Results and Discussion}

\section{Conservation of NRPS gene cluster across Teredinibacter turnerae}

A previous systematic analysis of shipworm symbiont isolate genomes and animal gill metagenomes identified a NRPS BGC, where it was defined as "GCF_1", as being highly conserved in T. turnerae, and portions of the cluster were detected in all T. turnerae-containing shipworms. ${ }^{\left[{ }^{8]}\right.}$ Here, multigene BLAST analysis of 8 sequenced T. turnerae strains found this cluster to be ubiquitous (Figure 1A); however, the region including turD and turE appeared to be regularly disrupted in many of these strains. In correctly assembled sequences, this region contained two repeats of $6 \mathrm{k}$ bp in length that were $98 \%$ identical, while in the misassembled sequences this region was scrambled. Therefore, it is likely that this is a result of bioinformatic assembly error, and the gene cluster is intact and functional in all strains. All of the intact NRPSs were further compared by BLASTp, and it was found that each megasynthetase was greater than $85 \%$ similar to all of the other corresponding proteins from the homologous clusters, demonstrating a very high degree of conservation (Figure 1B).

In the T. turnerae T7901 genome, the five genes within this cluster include turA ( 3 modules, $11.2 \mathrm{kbp}$ ), turB ( 3 modules, $12.8 \mathrm{kbp}$ ), turC ( 2 modules, $6.6 \mathrm{kbp}$ ), turD (2 modules, $8.1 \mathrm{kbp}$ ), and turE ( 3 modules, $12.1 \mathrm{kbp}$ ). Each module contains a condensation (C), adenylation $(\mathrm{A})$, and thiolation $(\mathrm{T})$ domain, and 5 of the modules also contain epimerization domains. The first domain begins with a so called $\mathrm{C}$ starter domain, which are known to acylate the initial amino acid in a process known as lipoinitiation. ${ }^{[13]}$ The final module terminates in a thioesterase domain, which is responsible for product release, often by means of macrocyclization. ${ }^{[14]}$ This domain architecture indicates that the product of the tur cluster is a cyclic lipopeptide, a known class of antibacterial scaffolds.

Often, NRPS genes are naturally combinatorialized to create families of compounds that consist of structural analogs distributed throughout related producing organisms. ${ }^{[15]}$ In order to look for the potential of variants with divergent peptide backbones, the adenylation domains for the cluster in each of the 8 sequenced strains were compared by BLASTp. In some of the misassembled 
sequences, the A domains in turD and turE were not intact, and were thus not included in the analysis. The results show that tur is an exceptionally well-conserved cluster, with each A domain retaining greater than $90 \%$ identity to all of the analogous domains across all 8 strains (Figure 1C). The substrate prediction, as determined by AntiSmash 5.0, was also consistent for each domain. Importantly, the strains included in this analysis were isolated from a variety of shipworm genera and species collected in disparate locations around the world, indicating that this gene cluster, and thus its precise biosynthetic product, are extremely well preserved, and thus likely important to the association between host and symbiont (Table S5) 

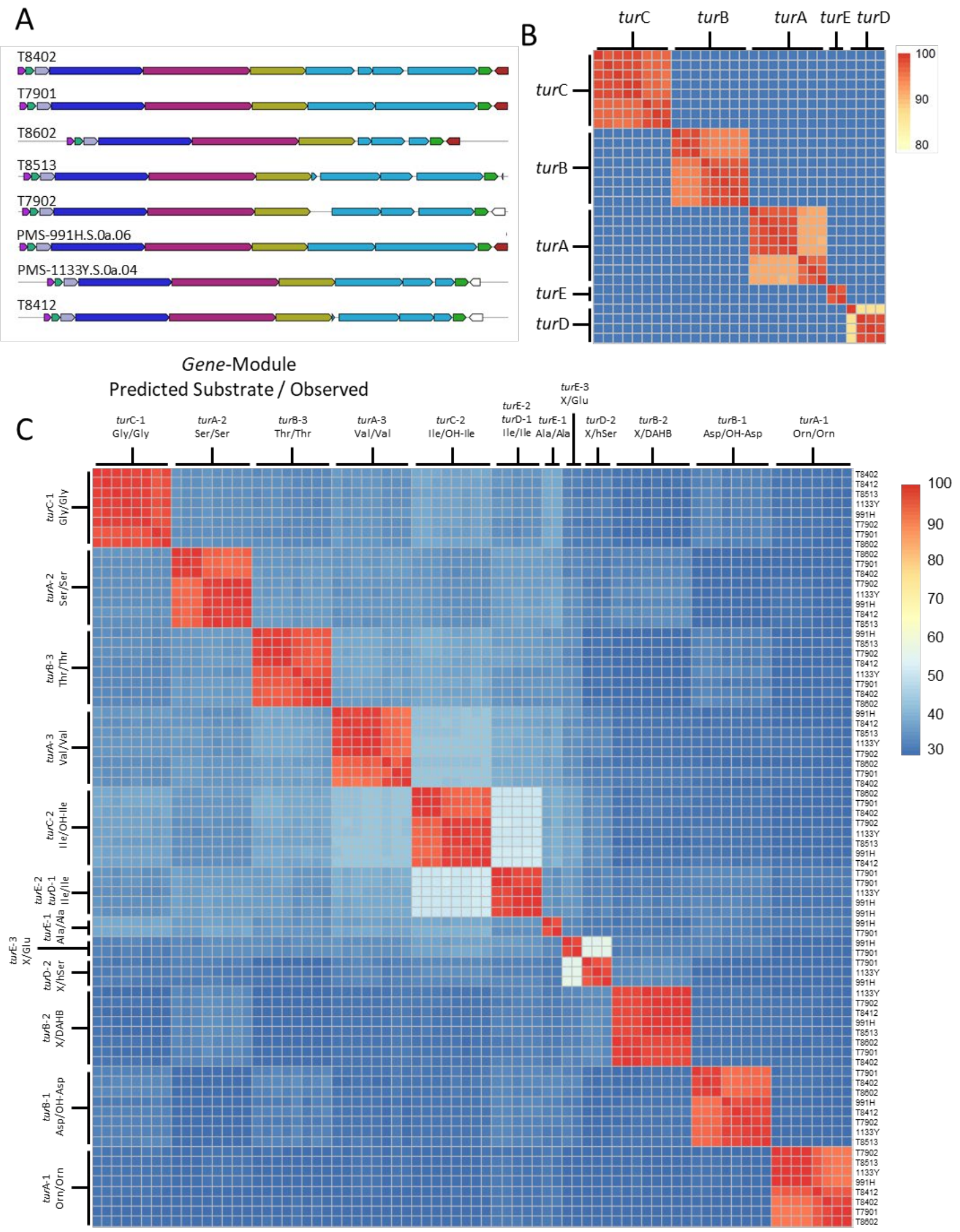

Figure 1. A) Multigene BLAST identified a conserved NRPS cluster present in eight sequenced T. turnerae strains. B) The proteins encoded by each NRPS gene are greater than $85 \%$ identical when compared to corresponding proteins in all eight clusters. C) BLASTp identity comparison of extracted adenylation domains from all identified tur gene clusters. Corresponding adenylation domains retain greater than $90 \%$ identity, indicating that the peptide backbone of the product is likely identical across all $T$. turnerae strains. 
Based upon the ubiquitous nature of this potential antibiotic, which would function in the symbiosis to defend the host and symbiont from other bacteria, we prioritized discovering the products of the tur pathway. A liquid culture of T. turnerae T7901 (6.6L) was extracted and subjected to a series of partitions. A particularly robust boundary layer formed between the water and ethyl acetate fractions, which was enriched in a series of lipopeptides. Semi-preparative HPLC yielded two major compounds $\mathbf{1}$ and $\mathbf{2}$, along with minor analogs $\mathbf{3}$ and 4.

Turnercyclamycin $A$ (1) had a molecular formula of $\mathrm{C}_{71} \mathrm{H}_{125} \mathrm{~N}_{15} \mathrm{O}_{24}$ based upon high resolution mass spectrometry $\left([\mathrm{M}+2 \mathrm{H}]^{2+}\right.$ $\mathrm{m} / \mathrm{z}=786.9618$ ), inherent in which are 17 degrees of unsaturation. The ${ }^{1} \mathrm{H}$ and ${ }^{13} \mathrm{C}$ spectra were consistent with a lipopeptide based on numerous amide $\mathrm{NH}$ resonances between $\delta \mathrm{H}$ 7.6-8.5, alpha proton signals from $\delta \mathrm{H}$ 4.03-5.05, and the presence of a large methylene envelope centered at $\delta \mathrm{H} 1.23$. The gHSQC experiment revealed the presence of 11 methyl groups, 17 distinct methylene proton pairs outside of the lipid envelope, and 19 methine protons (Table S1)

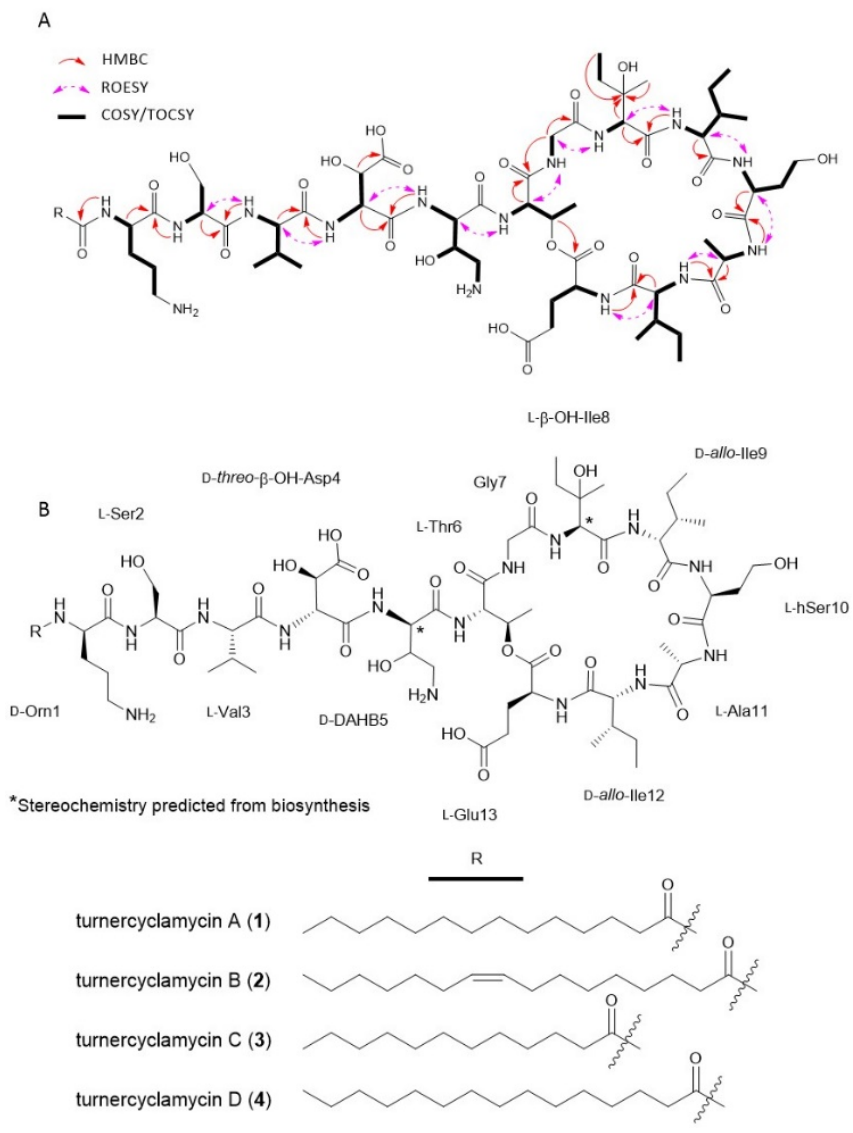

Figure 2: Structure elucidation of turnercylamycins A-D. (A) COSY/TOCSY (bold bonds), key ROESY (purple, dashed arrows), and key HMBC (red, solid arrows) correlations used to solve the planar structure. (B) Structures of turnercyclamycins with assigned absolute configurations. Red amino acid labels indicate D-configuration, green indicates L-configuration, and blue hydroxyl groups are proposed to be installed by tailoring enzymes.

The amide $\mathrm{NH}$ and alpha methine protons for eight amino acids could clearly be correlated through well separated gCOSY cross peaks, and verified by gHMBC experiments. A zTOCSY experiment further confirmed these eight correlations and identified an additional five residues. A combination of gCOSY, zTOCSY, and gHMBC experiments were used to elucidate the side chain from each alpha methine proton (Figure 2A), revealing the presence of one ornithine, one serine, one valine, one threonine, one glycine, two isoleucines, one glutamine, one alanine, one homoserine, one $\beta$-hydroxyaspartic acid, one $\beta$-hydroxyisoleucine, and an intriguing 2,4-diamino-3hydroxybutanoic acid (DAHB) moiety. The presence of all amino acids was confirmed through subsequent Marfey's analysis, which also revealed masses consistent with the FDLA-derivatized $\beta$-hydroxyisoleucine and DAHB moieties, despite the lack of standards for retention time comparison.

gHMBC correlations from amide $\mathrm{NH}$ protons to carbonyls, alpha methine protons to carbonyls, and ROESY correlations from amide $\mathrm{NH}$ protons to alpha methine protons were used to determine the sequence of residues in the peptide portion of the molecule. These data converged on the linear sequence of Orn-Ser-Val- $\beta-\mathrm{OH}-\mathrm{Asp}-\mathrm{DAHB}-\mathrm{Thr}-\mathrm{Gly}-\beta-\mathrm{OH}-\mathrm{Ile}-\mathrm{Ile}-\mathrm{hSer}-\mathrm{Ala}-\mathrm{Ile}-\mathrm{Glu}$, which was consistent with the order of substrate predictions for the adenylation domains of each NRPS module in the proposed biosynthetic pathway. 
The formula of the peptide backbone left a remainder of $\mathrm{C}_{14} \mathrm{H}_{21} \mathrm{O}$, indicating a tetradecanoic acid, and two degrees of unsaturation. TOCSY and gHMBC correlations verified the fatty acid moiety, and gHMBC and ROESY correlations confirmed it was linked through the terminal ornithine residue. The final remaining degree of unsaturation indicated macrocylization, a common feature of lipodesipeptides. A key HMBC correlation from the $\beta-H$ of the threonine residue $\left(\delta_{H} 5.06\right)$ to the carbonyl of the glutamic acid moiety $\left(\delta_{c} 170.7\right)$ indicated that macrocyclization is via ester linkage through the threonine oxygen. This was further supported by the downfield shifted $\beta$-oxymethine carbon at $\delta_{\mathrm{c}} 70.2$.

Turnercyclamycin B (2) was obtained as the second major compound. Based on the observed $[\mathrm{M}+2 \mathrm{H}]^{2+}$ ion at $m / z=799.9693$, its molecular formula was $\mathrm{C}_{73} \mathrm{H}_{127} \mathrm{~N}_{15} \mathrm{O}_{24}$, differing from that of 1 by $\mathrm{C}_{2} \mathrm{H}_{2}$ and an additional degree of unsaturation. Comparison of the proton and HSQC spectra showed that all of the chemical shifts and correlations associated with the peptide portion of the molecule were identical to those of 1 (Table S1). However, an HSQC correlation between $\delta_{H} 5.32\left(t, J=4.9 \mathrm{~Hz}, 2 \mathrm{H}\right.$ ) and $\delta_{C} 129.6$, as well as a distinct TOCSY spin system including a methylene envelop and the olefinic protons, indicated the presence of a cis- double bond within the fatty acid side chain.

To determine the location of the double bond in the lipid side chain of $\mathbf{2}$, ozonolysis was performed on intact $\mathbf{2}$ followed by a reductive workup and LCMS. A major detected $[\mathrm{M}+2 \mathrm{H}]^{2+}$ ion of $\mathrm{m} / \mathrm{z}=758.91$, corresponding to a monoisotopic mass of 1515.80 , matches the mass of the intact peptide with a 9-formyl-nonoic acid moiety (Figure S22). Therefore, 2 was determined to contain a cis-9hexadecanoic fatty acid tail.

The minor analogs turnercyclamycin C (3) and D (4) were obtained as pure compounds, but in insufficient yield for complete NMRbased structural characterization. However, the observed $[\mathrm{M}+2 \mathrm{H}]^{2+}$ ions at $\mathrm{m} / \mathrm{z}=772.9473$ for 3 and $\mathrm{m} / \mathrm{z}=793.9621$ for 4 indicated formulas of $\mathrm{C}_{69} \mathrm{H}_{121} \mathrm{~N}_{15} \mathrm{O}_{24}$ and $\mathrm{C}_{72} \mathrm{H}_{127} \mathrm{~N}_{15} \mathrm{O}_{24}$, respectively. The ${ }^{1} \mathrm{H}$ NMR and COSY spectra for both compounds are superimposable with those of 2 (Figures S17-S20). MSMS fragmentation localized the differences in mass to the lipid tail (Figure S23A-B), indicating that $\mathbf{3}$ and $\mathbf{4}$ are identical to $\mathbf{1}$ and $\mathbf{2}$, except that they contain unbranched C12:0 and C15:0 fatty acid tails, respectively.

A number of other minor analogs were identifiable by LCMS analysis, but were inseparable by HPLC. MSMS fragmentation revealed the inclusion of a number of other lipid tails on the same peptide core, as well as two structural modifications to the peptide portion of the molecule. One appears to be an ornithine to lysine substitution, which is well supported by multiple fragment ions (Figure S23D). The other is a loss of 14 amu that is localized to the cyclic portion of the peptide, but the fragmentation pattern did not clearly identify which amino acid was altered. The explanation most consistent with the data is that an isoleucine residue is substituted by valine.

Lastly, we detected by LCMS an assortment of analogs that represent ring-open linear peptides, methyl esters, and linear methyl esters. We propose that these represent hydrolysis artifacts and transesterification byproducts of the methanolic extraction and isolation work up (Table S3).

\section{Proposed biogenesis}

The adenylation domain substrate predictions of each NRPS module map very well to the NMR-elucidated structure. The only adenylation domains for which no substrate was predicted correspond to the incorporation of DAHB and homoserine, both unusual amino acids. The prediction for the modules incorporating $\beta-\mathrm{OH}$-Asp and $\beta-\mathrm{OH}$-lle were aspartic acid and isoleucine, respectively. Additionally, the presence or absence of epimerase domains perfectly matched the results of the Marfey's analysis, with $E$ domains present in all D-configuration amino acid extensions for which standards were available (Figure 3).

Upstream of the five NRPS megasynthase genes are four ORFs that are predicted to be involved in the tailoring and export of the compound. Two, turF and turH, encode alpha-ketoglutarate dependent dioxygenases (Fe/aKG), which are well characterized for performing hydroxylation reactions. ${ }^{[16]}$ Another, turG, encodes a cupin domain-containing protein with similarity to the protein JmjC. This class of enzyme also uses non-heme Fe and aKG to demethylate histones via hydroxylation, and related enzymes are responsible for the $\beta$-hydroxylation of lysine. ${ }^{[17,18]}$ It is likely that these three enzymes are performing the three $\beta$-hydroxylation reactions for the final product (Figure 3 ). 

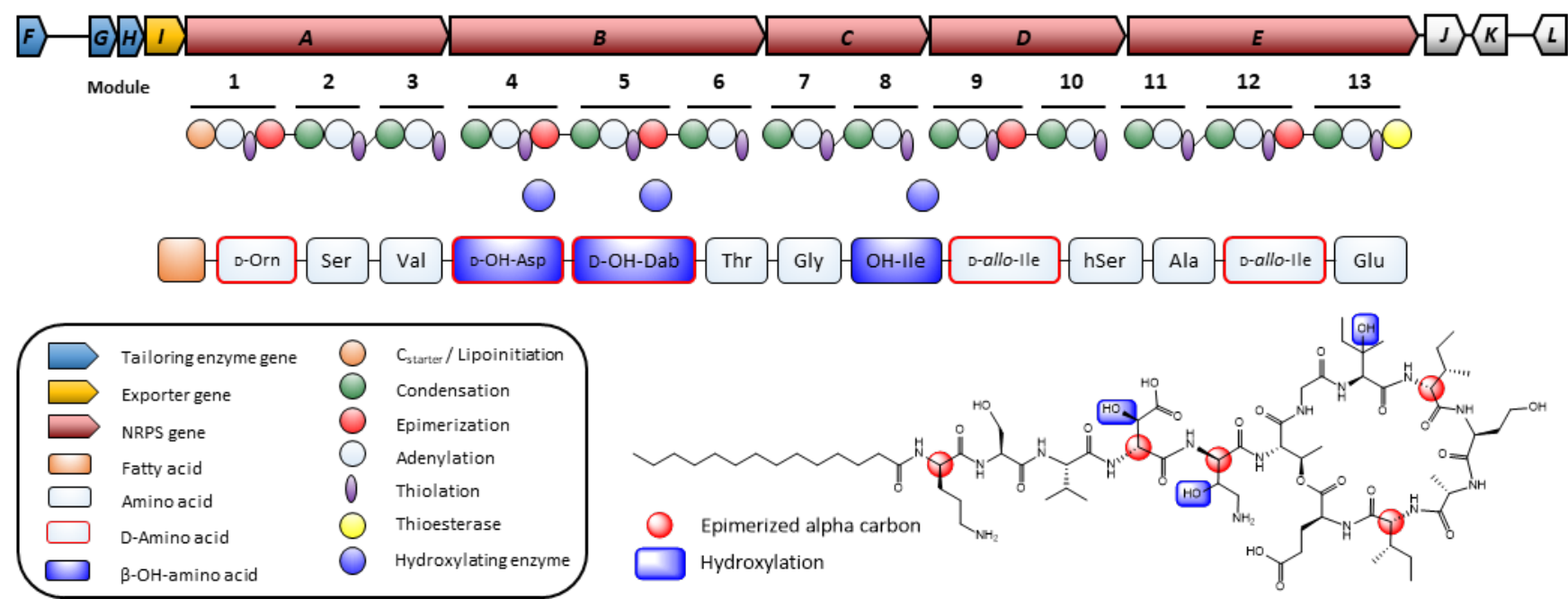

Figure 3. Proposed biogenesis

\section{Stereochemical assignment by advanced Marfey's analysis}

Compound 1 was hydrolyzed, and the constituent amino acids were derivatized with L-FDLA then analyzed by RP-UPLC-HRESIMS. The result was compared against derivatized standards, when available, and D-FDLA derivatized L-configuration standards were used to confirm retention times for $\mathrm{D}$-configuration amino acids. This analysis confirmed the serine, valine, threonine, homoserine, alanine, and glutamic acid residues are all L-configuration. The ornithine residue was determined to be in the D-configuration, and both isoleucine residues were confirmed to be D-allo-configured by comparison to D- and L-FDLA derivatized standards of L-Ile and L-allo-lle, which all separated by UPLC. These results were in accordance with the presence or absence of an epimerase domain within their associated NRPS modules in the proposed biosynthetic gene cluster.

The $\beta$-hydroxyaspartic acid was resolved based on comparison of the elution order to the reported values in Fuji et al ${ }^{[19]}$ and the elution pattern from the potashchalins. ${ }^{[20]}$ The hydrolysate of 1 was derivatized with both L-FDLA and D-FDLA, and after considerable LC method development, a small but distinct change in retention time was observed. The L-FDLA derivatized peak elutes earlier, which corresponds to the D-configured alpha carbon. This is further supported by the presence of an epimerase domain within the module incorporating this residue into the growing peptide chain. The L- and D-FDLA derivatized hydrolysate peaks were then compared to the L-FDLA derivatized D/L-threo- $\beta$-hydroxyaspartic acid standard. The elution of the standard matches well with that of the hydrolysate, and thus the absolute configuration is deemed to be D-threo- $\beta$-hydroxyaspartic acid. EICs and retention times for the derivitized hydrolaste and amino acid standards are supplied in Figure S21 and Table S2.

\section{Stereochemical prediction by biosynthetic logic}

The two remaining residues, $\beta$-hydroxyisoleucine and DAHB, both presented challenges due to the lack of any commercially available standards. $\beta$-hydroxyisoleucine is rarely found in natural products, and it was reported without stereochemical assignments in stalobactin ${ }^{[21]}$ and the $\mathrm{C} 2$ and $\mathrm{C} 3$ configurations of this residue were only resolved in phomopsin A through X-ray crystallography. ${ }^{[22]}$ Attempts to grow diffraction-quality crystals were unsuccessful, so investigation of the NRPS domain architecture for the proposed biosynthesis of the turnercyclamycins was used to predict the $\mathrm{C} 2$ stereochemistry. The L- or D-configuration of all ten residues that were assigned via Marfey's analysis perfectly correlated to the absence or presence of an epimerase domain. Thus, it is predicted that the $\beta$-hydroxyisoleucine is in the L- configuration due to the lack of an epimerase domain. The C3 position, however, remains ambiguous due to a lack of knowledge of the stereoselectivity of the hydroxylating enzyme for this position.

DAHB provides an additional challenge. This amino acid is found in several antibacterial lipopeptides, including odilorhabdins ${ }^{[23]}$ and ogipeptins ${ }^{[24]}$ but its biosynthesis remains unknown. Due to the common incorporation of DAHB in numerous cycliclipopetides and the presence of three separate enzymes capable of hydroxylating amino acids upstream of the NRPS cluster, we propose that this moiety is produced through the beta-hydroxylation of diaminobutyric acid (DAB). DAB is typically found in the L-configuration when an epimerase domain is not present in the NRPS module, so the presence of an epimerase domain in the module responsible for its incorporation indicates a D- configuration at position C2. DAHB is also found in peptidyl nucleic acid antibiotics, where it is made by a different biosynthetic route. 
Table 1. MICs of 1 and 2 bacterial and human (HEK-293) cells

\begin{tabular}{lll} 
Pathogen or Cell Line & \multicolumn{2}{l}{ MIC $(\mu \mathrm{g} / \mathrm{mL})$} \\
\cline { 2 - 2 } & $\mathbf{1}$ & $\mathbf{2}$ \\
\hline Acinetobacter baumannii & 8 & 8 \\
Escherichia coli & 1 & 1 \\
Enterococcus faecalis & $>64$ & $>64$ \\
Klebsiella pneumoniae & 2 & 2 \\
Staphylococcus aureus & $>64$ & $>64$ \\
HEK-293 & $>64$ & $>64$
\end{tabular}

Compounds 1 and $\mathbf{2}$ were initially tested against a panel of Gram-positive and Gram-negative pathogens in liquid broth assays. Both 1 and 2 were significantly active against Escherichia coli (MIC $1 \mu \mathrm{g} / \mathrm{mL}$ ), Acinetobacter baumannii (MIC $8 \mu \mathrm{g} / \mathrm{mL}$ ), and Klebsiella pneumoniae (MIC $2 \mu \mathrm{g} / \mathrm{mL}$ ), and neither compound showed activity against Enterococcus faecalis up to $64 \mu \mathrm{g} / \mathrm{mL}$ (Table 1). Neither 1 nor 2 were bactericidal to Staphylococcus aureus at concentrations up to $64 \mu \mathrm{g} / \mathrm{mL}$, but at $4 \mu \mathrm{g} / \mathrm{mL}$ and above they slowed growth, decreasing it by $50 \%$ during the period of the assay (Figure S24A). Since many antibiotics are relatively inactive against $A$. baumannii, we further investigated this activity, substituting the nonpathogenic relative $A$. baylyi. 1 and $\mathbf{2}$ showed the same MIC against $A$. baylyi, with similar phenotypic effects on the organism, validating the use of the strain. Plating of inhibited wells from $A$. baylyi broth cultures determined that the growth inhibition was a result of bactericidal, not bacteriostatic, activity (Figure S26).

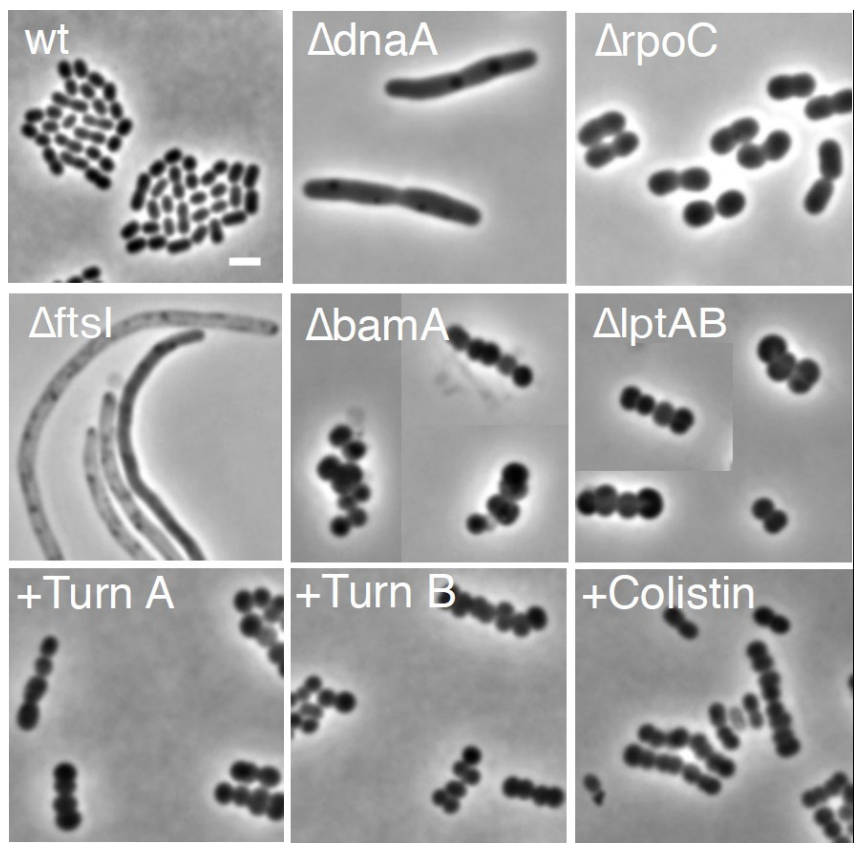

Figure 4. Terminal morphologies of mutant and antibiotic-treated $A$. baylyi. The images show bacteria whose growth has been inhibited by essential gene deletion or treatment with antibiotics at approximately the MICs (turnercyclamycin A and B, $8 \mu \mathrm{g} / \mathrm{ml}$; colistin, 0.5 $\mu \mathrm{g} / \mathrm{ml}$ ). The deletion mutations inactivate the following processes: dnaA, DNA replication; rpoC, transcription; ftsl, cell division; bamA, outer membrane protein localization; IptAB, LPS localization. Scale bar, $2 \mu \mathrm{m}$. wt, wild-type; TurnA, turnercyclamycin A (1); Turn $\mathrm{B}$, turnercyclamycin B (2).

Lipopeptide antibiotics often meet hurdles in development due to toxicity against mammalian cells and the lysing of erythrocytes owing to their amphipathic nature. ${ }^{[25]}$ Thus, $\mathbf{1}$ and $\mathbf{2}$ were tested for cytotoxicity against the human kidney-derived HEK-293 cell line and for hemolytic activity in freshly harvested murine erythrocytes. Both compounds showed no activity in either assay up to $64 \mu \mathrm{g} / \mathrm{mL}$, in comparison to control compounds that behaved as expected.

The combination of bactericidal activity against Acinetobacter and a lack of observed toxicity led us to further investigate the mechanism of action. A phenotypic assay was employed in which $A$. baylyi was cultivated in the presence or absence of drug, and the morphology and growth characteristics were compared to knockout mutants that are deficient in various essential proteins. ${ }^{[26,27]} \mathrm{A}$ total of 43 genes corresponding to 14 essential processes have been deleted, and the terminal morphologies of the mutant cells are distinct and depend on the process inactivated (Figure $\mathbf{4}$ and data not shown). In the presence of either $\mathbf{1}$ or $\mathbf{2}$ at the MIC, cells would divide a few times, then arrest growth as clumps of rounded cells. This death phenotype was strikingly similar to what is observed in mutants deficient in outer membrane protein and LPS synthesis (bamA, IptAB, $\operatorname{tam} A B$, and $I O / C D$ ) (Figure 4). Treatment with colistin, a clinically used 
lipopeptide targeting the outer membrane, also produced clumped, rounded cells. At concentrations 4 times the MIC, both 1 and 2 killed cells immediately and without the same morphological changes, potentially indicating a second mechanism at higher concentrations. Overall, the findings imply that the turnercyclamycins compromise outer membrane integrity.

If colistin and turnercyclamycins have the same molecular mechanism, it might be expected that colistin-resistant strains would also evade turnercyclamycins. To test this, we obtained clinical Acinetobacter baumannii complex (ABC) isolates from the Associated Regional and University Pathologists (ARUP) laboratory and the A. baumannii panel from the Centers for Disease Control and Prevention (CDC). We tested 6 of the CDC strains, including 2 each of strains susceptible, moderately resistant, and highly resistant to colistin. The 3 ARUP strains also showed a range of colistin resistance levels. Strikingly, turnercyclamycins retained their potency against these strains, including against highly colistin-resistant Acinetobacter strains (Table 2). This implies that the molecular mechanisms of these two lipopeptide classes may differ.

Table 2: MICs against ARUP and CDC panels of Acinetobacter strains with varying degrees of resistance against colistin.

\begin{tabular}{llll} 
Pathogen & \multicolumn{3}{c}{$\mathrm{MIC}(\mu \mathrm{g} / \mathrm{mL})$} \\
\cline { 2 - 3 } & $\mathbf{1}$ & $\mathbf{2}$ & Colistin \\
\hline Acinetobacter baumannii & 8 & 8 & 2 \\
ARUP $A B C 1$ & 4 & 4 & 4 \\
ARUP $A B C 2$ & 8 & 8 & 4 \\
ARUP $A B C 3$ & 16 & 8 & $>32$ \\
CDC $A B 282$ & 4 & 4 & 1 \\
CDC $A B 286$ & 4 & 4 & 1 \\
CDC $A B 299$ & 2 & 4 & 2 \\
CDC $A B 302$ & 8 & 4 & 2 \\
CDC $A B 303$ & 8 & 8 & $>32$ \\
CDC $A B 307$ & 8 & 8 & 8
\end{tabular}

\section{Conclusion}

Antimicrobial compounds, particularly those with new structural scaffolds and potentially new mechanisms of action, are in high demand. Each year, at least 35,000 people die from antibiotic-resistant bacterial infections in the United States. ${ }^{[28-31]}$ This is a number that keeps increasing, as bacteria are increasingly resistant to available drugs. Acinetobacter is an especially daunting pathogen, with more than $60 \%$ of patient-isolated strains showing resistance to standard treatments derived from beta-lactams or fluoroquinolones. An increasing number of strains is extremely drug-resistant, being untreatable even by the last-line lipopeptide agent, colistin (polymyxin E). Because there are very few Acinetobacter treatments in development, the US Centers for Disease Control considers carbapenem-resistant Acinetobacter to be an urgent threat. ${ }^{[32]}$ Here, we describe a new class of lipopeptides that are still effective against colistin-resistant Acinetobacter.

Symbiotic relationships represent unique environments that are promising for antimicrobial natural products discovery. These systems are typically described and understood based on the primary metabolic and nutritional interactions involved. However, exchanges of secondary metabolites also play important roles in these relationships. Microbes living within animal host tissues are of particular interest for antibiotic discovery because compounds made within these contexts must be non-toxic to the host cells and possess properties that facilitate distribution throughout the host tissues, which can translate to favorable pharmacokinetics. The shipwormTeredinibacter symbiotic relationship was investigated as a particularly promising source of antibiotic compounds due to: 1) previous studies showing a nearly axenic cecum, despite this being the site of cellulose degradation, likely resulting in an abundance of free glucose; 2 ) the presence of bacterial cellulases in this same organ, suggesting a route of transport from symbiont-containing gills to the cecum; and 3) a number of highly conserved biosynthetic gene clusters shared throughout the Teredinibacter lineage that could be producing complex PKS and NRPS compounds.

Following our ecology-guided rationale, a new family of cyclic lipodepsipeptides, the turnercyclamycins, was isolated and found to have activity against multiple Gram-negative pathogens including colistin resistant Acinetobacter. Last-line antibiotic resistance is a widespread problem beyond Acinetobacter, including in $E$. coli and other Gram-negative pathogens, so with further study this new family of lipopeptides may find an impact on therapy. This family consists of a conserved, NRPS-derived 13 amino acid core sequence and a variable fatty alkyl tail. The peptide portion is cyclized through an ester linkage via the threonine hydroxyl group, resulting in an eight amino acid macrocycle and five amino acid linear portion. A number of features are unique to this structural family, most notably the inclusion of three $\beta$-hydroxy amino acid residues, two of which, $\beta$-hydroxyisoleucine and 2,4-diamino-3-hydroxy-butanoic acid, are exceptionally rare. There is precedent for $\beta$-hydroxyaspartic acid in the literature, as it is a common feature of microbial siderophores. 
Genes encoding three enzymes likely to perform these hydroxylation reactions are found upstream of the NRPS megasynthases. Two of these, turF and turH, encode non-heme Fe(II)/aKG dependent dioxygenases, while the third, turG, encodes a cupin domaincontaining protein that has similarity to the $\mathrm{JmjC}$ family enzymes, which include hydroxylases and demethylases. While it is not currently known which enzyme is responsible for the hydroxylation of each residue, literature precedent and an examination of stereochemistry and domain architecture can give some clues. A recent genomic analysis of aspartyl $\beta$-hydroxylases identified two subtypes, one standalone $\mathrm{Fe}(\mathrm{II}) / \mathrm{aKG}$ enzyme and one encoded within the NRPS domain. ${ }^{[33]}$ All of the known standalone hydroxylases act on the PCPbound amino acid and produce either the D-threo or L-erythro stereoisomer. The presence of D-threo- $\beta$-hydroxyaspartic acid in the turnercyclamycins suggests that either turF or turH is acting in this capacity. While the same analysis identified 31 absolutely conserved residues in all previously describe aspartyl hydroxylases, neither turF nor turH contains all 31. However, turH does contain 29/31 conserved residues, and aligns more closely with the sequences of the aspartyl hydroxylases, and is thus a good candidate for this particular tailoring reaction.

It is unclear how the other two hydroxylated amino acids are formed. Due to the high prevalence of DAB moieties in lipopeptides, it is feasible that $D A B$ is first incorporated into the growing chain, then hydroxylated while bound to the thiolation domain, similar to the aspartic acid. However, there is precedent for post-translational $\beta$-hydroxylation of lysine residues on proteins by $\mathrm{JmjC}$ oxygenases. ${ }^{[18]}$ BLAST analysis of turG showed a conserved JmjC-like domain, so this possibility cannot be ruled out. Finally, there is literature precedent for the hydroxylation of free amino acids, including isoleucine, by Fe(II)/aKG dependent dioxygenases. ${ }^{[34]}$ While the adenylation domains in the modules responsible for incorporating the two lle residues are almost identical, they only share approximately $55 \%$ identity to the A domain of turC that incorporates $\mathrm{OH}-\mathrm{lle}$. It is feasible that one of the dioxygenases is acting upon free lle to produce a local pool of $\beta-\mathrm{OH}-l l e$, which is then recognized as the substrate for the A domain and incorporated into the growing chain.

The chemical properties of the turnercyclamycins are also intriguing when considering their mechanism of action, especially in relation to colistin. Both compounds are cyclic lipopeptides that affect outer membrane integrity, but the Acinetobacter strains with resistance to colistin are still susceptible to turnercyclamycins. It is believed that colistin works largely due to the highly cationic peptide portion, which gains its positive charges through the side chains of five DAB moieties. This charged peptide displaces magnesium and calcium counter ions in the LPS, while the lipid tail works to solubilize the membrane through a detergent-like mechanism. ${ }^{[35]}$ The turnercyclamycins, on the other hand, are not rich in DAB residues or other cationic peptide side chains, and indeed maintain a neutral charge balance. This chemical property may be important in explaining how the turnercyclamycins retain activity against colistin resistant strains, and likely points to a different molecular mechanism of action that accomplishes the same goal of outer membrane disruption.

Secondary metabolism plays a large role in the association between $T$. turnerae and their shipworm hosts. Thus far, of the ubiquitous biosynthetic pathways found in all $T$. turnerae strains and in all shipworm gill metagenomes in which $T$. turnerae is a symbiont, three have been characterized. Because of their ubiquity, all three likely play crucial roles in symbiosis biology. The exquisite potency of tartrolon D/E against apicomplexan parasites is hypothesized to protect the mollusk from gregarines, which are known to be pathogens of mollusks. ${ }^{[11,36]}$ Turnerbactin, the triscatecholate siderophore, may be important both for the acquisition of iron for the symbiont and host, as well as for the sequestration of iron to limit the growth of pathogenic or opportunistic bacteria. ${ }^{\left[{ }^{9]}\right.}$ This most recent discovery of the turnercyclamycins represents the addition of a potent bactericidal agent to the molecular arsenal of $T$. turnerae, capable of directly killing bacteria that pose threats to its shipworm host, their shared food supply, or the ecological niche of the symbiont itself. This finding underscores the importance of closely examining the chemical biology of symbiotic systems with a particular focus on ecological rationale and metagenomics in the pursuit of new pharmacological agents for improved human health.

\section{Experimental Procedures}

\section{General Experimental Procedures}

UV-vis spectra were obtained using a Molecular Devices SpectraMax M2 spectrophotometer. High resolution mass spectra were acquired using a Waters Xevo G2-XS QTof mass spectrometer equipped with a Zspray ESI source and fed by an Acquity $\mathrm{H}$ class UPLC system with a Waters Acquity CSH C18 column $(2.1 \times 50 \mathrm{~mm}, 1.8 \mathrm{um})$. NMR data were collected using a Varian $500 \mathrm{MHz}$ NMR spectrometer with $5 \mathrm{~mm}$ Varian HCN Oneprobe for proton detected experiments and a $3 \mathrm{~mm}$ Varian inverse probe for carbon detected experiments $\left({ }^{1} \mathrm{H} 500 \mathrm{MHz},{ }^{13} \mathrm{C} 125 \mathrm{MHz}\right)$. Residual signals from solvents were used for referencing. ECD spectra were obtained on an AVIV Biomedical, Inc. CD Spec Model 410 (Lakewood, NJ, USA). Analytical and semi-preparative HPLC was performed on a Thermo UltiMate 3000 system with a DAD detector.

\section{Fermentation, chemical extraction, and purification}

A glycerol stock of $T$. turnerae T7901 was revived by streaking $5 \mu \mathrm{L}$ on a plate of Shipworm Basal Medium (SBM). ${ }^{[3]} 5 \mathrm{~mL}$ liquid SBM cultures were inoculated with single colonies and incubated at $30{ }^{\circ} \mathrm{C}$ with shaking at $180 \mathrm{RPM}$ for 4 days. These seed cultures were used to inoculate six liquid SBM (with phosphate $\mathrm{f} / \mathrm{c}=15 \mu \mathrm{M}$ ) $1.1 \mathrm{~L}$ cultures in $2.8 \mathrm{~L}$ baffled Fernbach flasks, which were incubated at $30^{\circ} \mathrm{C}$ with shaking at $180 \mathrm{RPM}$ for 7 days. Cells were removed from culture media by centrifugation at $7,068 \times \mathrm{g}, 4^{\circ} \mathrm{C}$, for $30 \mathrm{~min}$. The supernatant was decanted, and the cell pellet was frozen and lyophilized to dryness. 
The dry cell pellet was extracted three times with $400 \mathrm{~mL}$ each of 2:1 DCM:MeOH. The extracts were combined and concentrated in vacuo to remove DCM, and water was added to a composition of $5 \% \mathrm{v} / \mathrm{v}$ in $\mathrm{MeOH}$, then partitioned 3 time with an equal volume of hexanes. The methanolic fraction was dried in vacuo, then partitioned 3 times between equal volumes of water and DCM. The aqueous layer was then subsequently partitioned 3 times with equal volumes of ethyl acetate. This step formed a particularly well-defined insoluble boundary layer, which was collected separately.

The boundary layer was dried in vacuo then resuspended in $\mathrm{MeOH}$. This was centrifuged to pellet insoluble material, then the supernatant was filtered (Nylon, $0.45 \mu \mathrm{m}$ ) and subjected to semipreparative RPHPLC (Phenomenex Luna $\mathrm{C}_{18}$ column, $250 \times 10 \mathrm{~mm}, 5$ $\mu \mathrm{m}$, flow rate $4.0 \mathrm{~mL} / \mathrm{min})$. Isocratic conditions $\left(20 \% \mathrm{ACN} / 80 \% \mathrm{H}_{2} \mathrm{O} / 0.01 \%\right.$ TFA) were held for 5 min followed by a linear gradient to $75 \% \mathrm{ACN} / 25 \% \mathrm{H}_{2} \mathrm{O} / 0.01 \%$ TFA for $15 \mathrm{~min}$ to yield turnercyclamycins $\mathrm{A}-\mathrm{D}\left(\mathbf{1}, t_{\mathrm{R}}=15.3 \mathrm{~min}, 5.6 \mathrm{mg} ; \mathbf{2}, t_{\mathrm{R}}=15.8 \mathrm{~min}, 6.8 \mathrm{mg} ; \mathbf{3}, t_{\mathrm{R}}=\right.$ $14.2 \mathrm{~min}, 0.9 \mathrm{mg} ; 4, t_{\mathrm{R}}=16.8 \mathrm{~min}, 0.6 \mathrm{mg}$ ).

Turnercyclamycin A (1):

Off-white amorphous solid; UV/vis: $\lambda_{\max } 250,270 \mathrm{~nm}$; IR (neat) $v_{\max } 3296,3067,2930,1645,1538,1204,1140 \mathrm{~cm}^{-1} ;{ }^{1} \mathrm{H}$ and ${ }^{13} \mathrm{C} \mathrm{NMR}$ data in DMSO- $d_{6}$, Table 1; HRESIMS $[\mathrm{M}+2 \mathrm{H}]^{2+}$ ion at $m / z=786.9619$ (calculated for $\mathrm{C}_{71} \mathrm{H}_{125} \mathrm{~N}_{15} \mathrm{O}_{24}: 786.9584$ )

Turnercyclamycin B (2):

Off-white amorphous solid; UV/vis: $\lambda_{\max } 250,270 \mathrm{~nm}$; IR (neat) $\mathrm{V}_{\max } 3296,3065,2921,1645,1540,1204,1139 \mathrm{~cm}^{-1} ;{ }^{1} \mathrm{H}$ and ${ }^{13} \mathrm{C} \mathrm{NMR}$ data in DMSO- $d_{6}$, Table 1; HRESIMS $[\mathrm{M}+2 \mathrm{H}]^{2+}$ ion at $m / z=799.9693$ (calculated $\mathrm{C}_{73} \mathrm{H}_{127} \mathrm{~N}_{15} \mathrm{O}_{24}=799.9662$ )

Turnercyclamycin C (3):

Off-white amorphous solid; ${ }^{1} \mathrm{H}$ NMR in DMSO- $d_{6}$, Figure S17; HRESIMS $[\mathrm{M}+2 \mathrm{H}]^{2+}$ ion at $m / z=772.9473$ (calculated for $\mathrm{C}_{69} \mathrm{H}_{121} \mathrm{~N}_{15} \mathrm{O}_{24}$ : 772.9427)

Turnercyclamycin D (4):

Off-white amorphous solid; ${ }^{1} \mathrm{H}$ NMR in DMSO- $d_{6}$, Figure S19; HRESIMS $[\mathrm{M}+2 \mathrm{H}]^{2+}$ ion at $m / z=793.9621$ (calculated for $\mathrm{C}_{72} \mathrm{H}_{127} \mathrm{~N}_{15} \mathrm{O}_{24}$ : 793.9662)

\section{Determination of amino acid stereochemistry}

$1 \mathrm{~mL} 6 \mathrm{~N} \mathrm{HCl}$ was added separately to $1 \mathrm{mg}$ of 1 and $1 \mathrm{mg}$ of 2 and the reaction vessels were heated to $100{ }^{\circ} \mathrm{C}$ with stirring for $24 \mathrm{~h}$. Solvent was evaporated under a stream of nitrogen gas and the resulting residues were dissolved in $250 \mu \mathrm{L}$ of $\mathrm{H}_{2} \mathrm{O}$. Aliquots $(50 \mu \mathrm{L})$ of each hydrolysate solution were transferred to a clean glass vial, to which $20 \mu \mathrm{L}$ aqueous $1 \mathrm{M} \mathrm{NaHCO}_{3}$ and $100 \mu \mathrm{L}$ Marfey's reagent (L-FDLA, $1 \%$ solution in acetone, TCI Chemicals) were added. The reactions were incubated for $1 \mathrm{~h}$ at $40{ }^{\circ} \mathrm{C}$, then quenched with 20 $\mu \mathrm{L} 1 \mathrm{~N} \mathrm{HCl}$. This solution was filtered through a polypropylene filter $(0.45 \mu \mathrm{m})$, then diluted into $\mathrm{MeOH}$ for UPLC-MS analysis. Analysis was performed on an analytical Waters Acquity ${ }^{\circledR}$ UPLC equipped with a Acquity ${ }^{\circledR}$ HSS T3 column $(100 \times 2.1 \mathrm{~mm}, 1.8 \mu \mathrm{m}$, Waters, flow rate $=0.3 \mathrm{~mL} \mathrm{~min}{ }^{-1}$, method: $0-2 \mathrm{~min}: 20 \%(\mathrm{v} / \mathrm{v}) \mathrm{ACN}$ in water containing $0.1 \%$ formic acid; $2-30$ min: linear gradient $20 \%-65 \%$ ACN in water containing $0.1 \%$ formic acid; $30-31$ min: linear gradient $65 \%-100 \%$ ACN in water containing $0.1 \%$ formic acid). Amino acid standards were derivatized in the same manner for comparison, and D-FDLA was used to derivatize L-configured amino acid standards to obtain the retention times for D-amino acids. Retention times for amino acids derived from 1, 2, and amino acids standards are summarized in table S1.

\section{Antimicrobial microdilution assay}

Glycerol stocks of Staphylococcus aureus (S. aureus subsp aureus ATCC $\left.{ }^{\circledR} 1600^{\mathrm{TM}}\right)$, Enterococcus faecalis (ATCC $\left.{ }^{\circledR} 29212^{\mathrm{TM}}\right)$, Klebsiella pneumoniae (ATCC $\AA$ BAA- $1705^{\mathrm{TM}}$ ), Acinetobacter baumannii (ATCC $\AA 19606^{\mathrm{TM}}$ ), and the CDC strains AB282, AB286, $A B 299$, and AB302 were streaked on Mueller Hinton Agar (MHA). ARUP Acinetobacter complex strains ABC1, ABC2, and ABC3, and $\mathrm{CDC}$ strains $\mathrm{AB} 303$ and $\mathrm{AB} 307$ were streaked on $\mathrm{MHA}$ including $2 \mu \mathrm{g} / \mathrm{mL}$ colistin to remove sensitive bacteria. Escherichia coli $\left(\right.$ ATCC $\otimes 23724^{\mathrm{TM}}$ ) was streaked on Luria-Bertani agar (LBA) plates. Plates were incubated at $37^{\circ} \mathrm{C}$ for $8-12 \mathrm{~h}$. Single colonies from the plates were then transferred into Mueller Hinton Broth II (MHB II) (Luria-Bertani broth for E. coli) and incubated for $6-8 \mathrm{~h}$ at $30{ }^{\circ} \mathrm{C}, 150$ $\mathrm{rpm}$. The turbidity of the broth culture was then adjusted to match $0.5 \mathrm{McF}$ arland standard $\left(1 \times 10^{8} \mathrm{cells} / \mathrm{mL}\right)$. The adjusted broth culture was diluted 200 -fold and used as inoculum for the assay. Each test organism $(200 \mu \mathrm{L})$ was added to each well of a 96 -well flat plate. Compounds were then added using a two-fold dilution scheme starting at $64 \mu \mathrm{g} / \mathrm{mL}$, with 8 dilutions each. Following $18-20 \mathrm{~h}$ incubation, MTT $(10 \mu \mathrm{L} ; 5 \mathrm{mg} / \mathrm{mL})$ was added to wells and incubated for $2 \mathrm{~h}$. DMSO $(100 \mu \mathrm{L})$ was then added to wells and incubated for $1 \mathrm{~h}$. The $\mathrm{A}_{570}$ was then measured using a Biotek-Synergy 2 Microplate Reader (Biotek).

\section{Mammalian antiproliferative assay}

HEK-293 (ATCC $® C R L-1573^{\text {TM }}$ ) cells were cultured in RPMI 1640 medium supplemented with $10 \%$ fetal bovine serum, 100 units of penicillin, and $100 \mu \mathrm{g} / \mathrm{mL}$ of streptomycin under a humidified environment with $5 \% \mathrm{CO}_{2}$ at $37{ }^{\circ} \mathrm{C}$. The cells were seeded $(10,000$ cells/well) in 96-well plates and treated after $24 \mathrm{~h}$ with varying concentrations of the compound. After $72 \mathrm{~h}$, the media was removed and MTT $(15 \mu \mathrm{L} ; 5 \mathrm{mg} / \mathrm{mL})$ was added to each well. The plates were then incubated for $3 \mathrm{~h}$ at $37{ }^{\circ} \mathrm{C}, 5 \% \mathrm{CO}_{2}$. After incubation, DMSO $(100 \mu \mathrm{L})$ was added and absorbance was read at $570 \mathrm{~nm}$ using a Biotek-Synergy 2 Microplate Reader (Biotek).

Hemolysis assay

Hemolytic activity of the compounds was measured using freshly purified $0.25 \%$ Cgrp grp mouse red blood cell suspension in $1 \mathrm{x}$ phosphate buffered saline (PBS). Various concentrations of the compounds were then added to the red blood cell suspension in 2-fold 
serial dilutions, and incubated for $1 \mathrm{~h}$ at $37^{\circ} \mathrm{C}$. The suspension was then centrifuged at $1000 \times g$ for 5 min, and the supernatant was collected. Supernatant $(100 \mu \mathrm{L})$ was then transferred to wells of a 96-well plate. Absorbance was read at $540 \mathrm{~nm}$ using a BiotekSynergy 2 Microplate Reader (Biotek). Results are reported as concentration of compound resulting in $10 \%$ hemolysis. Triton X-100 $(0.01-10 \% \mathrm{v} / \mathrm{v})$ served as positive control.

\section{Microscopy of growth-inhibited bacteria}

Acinetobacter baylyi strain ADP1 overnight cultures grown in M9 minimal-succinate medium were diluted 1:4 and grown with shaking for one additional hour at $30^{\circ} \mathrm{C}$., followed by spotting on thin minimal-succinate agar pads containing different drugs [turnercyclamycin $(8 \mu \mathrm{g} / \mathrm{mL})$ or colistin $(0.5 \mu \mathrm{g} / \mathrm{mL})]^{[27]}$ Bacteria were imaged after $8 \mathrm{~h}$ incubation at $30^{\circ} \mathrm{C}$. Terminal morphologies of essential gene deletion mutants were evaluated after transformation with kanamycin-resistance marked PCR fragments as described earlier. ${ }^{[26,27]}$ Phase contrast imaging was performed using a Nikon Eclipse 90 microscope using a 100x oil objective.

tur gene clusters analysis in Teredinibacter turnerae strains

A full or partial tur gene cluster was extracted from antiSMASH output of each $T$. turnerae genome assembly using multigeneblast with tur in T7901 as reference. Protein sequences of a full NRPS genes (according to "CDS" feature) or A-domains (according to "aSDomain" feature) were extracted from the antiSMASH output genbank file using a bash script (supplied below). The identity between NRPS genes or A-domains were calculated by BLASTp search (-outfmt "6 qseqid sseqid pident length evalue qcovs qlen slen"). An identity matrix table was made and plotted in R 3.5 using the pheatmap package.

\section{Acknowledgements}

We thank Xuchen Zhan and Jon Rainier (U. Utah) for assistance with ozonolysis and the Center for High Performance Computing, University of Utah for computational resources. This work was funded by grants U01TW008163 (NIH Fogarty) to MGH, NIH R35GM122521 to EWS, NOAA NA19OAR0110303 to EWS and MGH, and R01Al148208 to CM. We are grateful for key infrastructure support provided by the ALSAM Foundation.

Keywords: Antibiotics • Natural Products $・$ Nonribosomal Peptide $•$ Symbiosis $・$ Chemical Biology 


\section{References}

[1] D. L. Distel, D. J. Beaudoin, W. Morrill, Appl. Environ. Microbiol. 2002, 68, 6292-6299.

[2] N. A. Ekborg, W. Morrill, A. M. Burgoyne, L. Li, D. L. Distel, Appl. Environ. Microbiol. 2007, 73, 7785-7788.

[3] C. P. Lechene, Y. Luyten, G. McMahon, D. L. Distel, Science 2007, 317, 1563-1566.

[4] R. M. O'Connor, J. M. Fung, K. H. Sharp, J. S. Benner, C. McClung, S. Cushing, E. R. Lamkin, A. I. Fomenkov, B. Henrissat, Y. Y. Londer, M. B. Scholz, J. Posfai, S. Malfatti, S. G. Tringe, T. Woyke, R. R. Malmstrom, D. Coleman-Derr, M. A. Altamia, S. Dedrick, S. T. Kaluziak, M. G. Haygood, D. L. Distel, PNAS 2014, 111, E5096-E5104.

[5] M. A. Betcher, J. M. Fung, A. W. Han, R. O’Connor, R. Seronay, G. P. Concepcion, D. L. Distel, M. G. Haygood, PLOS ONE 2012, 7, e45309.

[6] S. I. Elshahawi, A. E. Trindade-Silva, A. Hanora, A. W. Han, M. S. Flores, V. Vizzoni, C. G. Schrago, C. A. Soares, G. P. Concepcion, D. L. Distel, E. W. Schmidt, M. G. Haygood, PNAS 2013, 110, E295-E304

[7] J. C. Yang, R. Madupu, A. S. Durkin, N. A. Ekborg, C. S. Pedamallu, J. B. Hostetler, D. Radune, B. S. Toms, B. Henrissat, P. M. Coutinho, S. Schwarz, L. Field, A. E. Trindade-Silva, C. A. G. Soares, S. Elshahawi, A. Hanora, E. W. Schmidt, M. G. Haygood, J. Posfai, J. Benner, C. Madinger, J. Nove, B. Anton, K. Chaudhary, J. Foster, A. Holman, S. Kumar, P. A. Lessard, Y. A. Luyten, B. Slatko, N. Wood, B. Wu, M. Teplitski, J. D. Mougous, N. Ward, J. A. Eisen, J. H. Badger, D. L. Distel, PLOS ONE 2009, 4, e6085.

[8] M. A. Altamia, Z. Lin, A. E. Trindade-Silva, I. D. Uy, J. R. Shipway, D. V. Wilke, G. P. Concepcion, D. L. Distel, E. W. Schmidt, M. G. Haygood, mSystems 2020, 5, DOI 10.1128/mSystems.00261-20.

[9] A. W. Han, M. Sandy, B. Fishman, A. E. Trindade-Silva, C. A. G. Soares, D. L. Distel, A. Butler, M. G. Haygood, PLOS ONE 2013, 8, e76151.

[10] J. M. Raaijmakers, L. van der Sluis, P. A. H. M. Bakker, B. Schippers, M. Koster, P. J. Weisbeek, Can. J. Microbiol. 1995, 41, 126-135.

[11] R. M. O'Connor, F. J. N. V, J. Abenoja, G. Bowden, P. Reis, J. Beaushaw, R. M. B. Relat, I. Driskell, F. Gimenez, M. W. Riggs, D. A. Schaefer, E. W. Schmidt, Z. Lin, D. L. Distel, J. Clardy, T. R. Ramadhar, D. R. Allred, H. M. Fritz, P. Rathod, L. Chery, J. White, PLOS Pathogens 2020, 16, e1008600. H. W. Boucher, G. H. Talbot, J. S. Bradley, J. E. Edwards, D. Gilbert, L. B. Rice, M. Scheld, B. Spellberg, J. Bartlett, Clin Infect Dis 2009, 48, 1-12.

[13] C. Rausch, I. Hoof, T. Weber, W. Wohlleben, D. H. Huson, BMC Evol Biol 2007, 7, 78.

[14] T. A. Keating, D. E. Ehmann, R. M. Kohli, C. G. Marshall, J. W. Trauger, C. T. Walsh, ChemBioChem 2001, 2, 99-107.

[15] J. Zan, Z. Li, M. D. Tianero, J. Davis, R. T. Hill, M. S. Donia, Science 2019, 364, DOI 10.1126/science.aaw6732.

[16] L.-F. Wu, S. Meng, G.-L. Tang, Biochimica et Biophysica Acta (BBA) - Proteins and Proteomics 2016, 1864, 453-470.

[17] Y. Tsukada, J. Fang, H. Erdjument-Bromage, M. E. Warren, C. H. Borchers, P. Tempst, Y. Zhang, Nature 2006, 439, 811-816.

[18] S. Markolovic, Q. Zhuang, S. E. Wilkins, C. D. Eaton, M. I. Abboud, M. J. Katz, H. E. McNeil, R. K. Leśniak, C. Hall, W. B. Struwe, R. Konietzny, S. Davis, M. Yang, W. Ge, J. L. P. Benesch, B. M. Kessler, P. J. Ratcliffe, M. E. Cockman, R. Fischer, P. Wappner, R. Chowdhury, M. L. Coleman, C. J. Schofield, Nat. Chem. Biol. 2018, 14, 688-695.

[19] K. Fujii, Y. Ikai, T. Mayumi, H. Oka, M. Suzuki, K. Harada, Anal. Chem. 1997, 69, 3346-3352.

[20] Y. Li, L. Liu, G. Zhang, N. He, W. Guo, B. Hong, Y. Xie, Front Chem 2020, 8, 197.

[21] K. Matsui, Y. Kan, J. Kikuchi, K. Matsushima, M. Takemura, H. Maki, I. Kozono, T. Ueda, K. Minagawa, J. Med. Chem. 2020, 63, 6090-6095.

[22] C. C. J. Culvenor, J. A. Edgar, M. F. Mackay, C. P. Gorst-Allman, W. F.O. Marasas, P. S. Steyn, R. Vleggaar, P. L. Wessels, Tetrahedron 1989, 45 2351-2372.

[23] L. Pantel, T. Florin, M. Dobosz-Bartoszek, E. Racine, M. Sarciaux, M. Serri, J. Houard, J.-M. Campagne, R. M. de Figueiredo, C. Midrier, S. Gaudriault, A. Givaudan, A. Lanois, S. Forst, A. Aumelas, C. Cotteaux-Lautard, J.-M. Bolla, C. Vingsbo Lundberg, D. L. Huseby, D. Hughes, P. Villain-Guillot, A. S. Mankin, Y. S. Polikanov, M. Gualtieri, Molecular Cell 2018, 70, 83-94.e7.

[24] S. Kozuma, Y. Hirota-Takahata, D. Fukuda, N. Kuraya, M. Nakajima, O. Ando, The Journal of Antibiotics 2017, 70, 79-83.

[25] G. Agner, Y. A. Kaulin, P. A. Gurnev, Z. Szabo, L. V. Schagina, J. Y. Takemoto, K. Blasko, Bioelectrochemistry 2000, 52, $161-167$.

[26] L. A. Gallagher, J. Bailey, C. Manoil, PNAS 2020, 117, 18010-18017.

[27] J. Bailey, J. Cass, J. Gasper, N.-D. Ngo, P. Wiggins, C. Manoil, PLOS Genetics 2019, 15, e1008195.

[28] E.-T. Piperaki, L. S. Tzouvelekis, V. Miriagou, G. L. Daikos, Clinical Microbiology and Infection 2019, 25, $951-957$.

[29] C.-R. Lee, J. H. Lee, M. Park, K. S. Park, I. K. Bae, Y. B. Kim, C.-J. Cha, B. C. Jeong, S. H. Lee, Front. Cell. Infect. Microbiol. 2017, 7, DOI 10.3389/fcimb.2017.00055

[30] D. Wong, T. B. Nielsen, R. A. Bonomo, P. Pantapalangkoor, B. Luna, B. Spellberg, Clinical Microbiology Reviews $2017,30,409-447$.

[31] A. Howard, M. O'Donoghue, A. Feeney, R. D. Sleator, Virulence 2012, 3, 243-250.

[32] Centers for Disease Control and Prevention (U.S.), Antibiotic Resistance Threats in the United States, 2019, Centers For Disease Control And Prevention (U.S.), 2019

[33] Z. L. Reitz, C. D. Hardy, J. Suk, J. Bouvet, A. Butler, PNAS 2019, 116, 19805-19814.

[34] M. Hibi, T. Kawashima, T. Kodera, S. V. Smirnov, P. M. Sokolov, M. Sugiyama, S. Shimizu, K. Yokozeki, J. Ogawa, Appl. Environ. Microbiol. 2011, 77, 6926-6930.

[35] R. A. Dixon, I. Chopra, Antimicrob Agents Chemother 1986, 29, 781-788.

[36] S. Rueckert, E. L. Betts, A. D. Tsaousis, Trends in Parasitology 2019, 35, 687-694.

[37] J. B. Waterbury, C. B. Calloway, R. D. Turner, Science 1983, 221, 1401-1403. 